\title{
EVIDENCE FOR THE EARTH'S GRAVITATIONAL TIME DELAY IN VLBI EXPERI- MENTS*
}

\author{
H.Schuh ${ }^{1}$, M.Fellbaum ${ }^{1}$, J.Campbell ${ }^{1}$, M.Soffel ${ }^{2,3}$, H.Ruder $^{2}$ and M.Schneider ${ }^{3}$ \\ ${ }^{1}$ Geodätisches Institut der Uni. Bonn, Nußallee 17 D-5300 Bonn 1, FRG \\ ${ }^{2}$ Lehrstuhl für Theor. Astrophysik, Auf der Morgenstelle 12, D-7400 Tübingen, \\ FRG \\ ${ }^{3}$ SFB78 Satellitengeodäsie, Technische Universität München, D-8000 München, FRG
}

\begin{abstract}
Evidence is found that in IRIS experiments the gravitational time delay due to the gravitational field of the Earth can be seen. Influences of this time delay upon baseline determinations are discussed.
\end{abstract}

\section{SUMMARY}

It seems to have been Carl Friedrich Gauss (1777 - 1855) who first considered the euclidean geometry of the space surrounding the Earth not to be self-evident but subject to operational verification. Apparently, he even surveyed a triangle in the Harz mountains formed by Inselberg, Brocken and Hoher Hagen to check whether the sum of a real triangle was indeed $180^{\circ}$. Later Einstein showed that, due to the validity of the equivalence principle, gravity can be understood as a phenomenon of a curved space-time where test particles (planets, photons, etc.) move on geodesics in the metric field that is determined by the energy momentum distribution in the universe. The light deflection in the gravitational field of the Sun is one important consequence of Einstein's theory of gravity which was first confirmed during the total eclipse in 1919. To post-Newtonian order the angle of light deflection is given by:

$$
\Delta \varphi=\left(\frac{\gamma+1}{2}\right) \frac{4 G m_{\odot}}{c^{2} d}
$$

where $d$ indicates the minimal distance of the light ray to the Sun and $\gamma$ is the space curvature parameter from PPN theory (e.g. Will 1981). Whereas optical light deflection measurements can determine the value for $\gamma$ with an accuracy of only $10 \%(\gamma=0.95 \pm 0.11$; Texas Mauritanian Eclipse Team 1976; Jones 1976) great progress was achieved here by radar time delay measurements (Reasenberg et al. 1979) and by VLBI measurements where the curvature of space-time leads to a gravitational time delay of radio signals of (e.g. Finkelstein et al. 1983, Soffel et al. 1986):

$$
\tau_{\mathrm{grav}}^{\odot}=\left(\frac{\gamma+1}{2}\right) \frac{2 G m_{\odot}}{c^{3}} \ln \left[\frac{\left|\mathbf{x}_{1}\right|+\mathbf{x}_{1} \cdot \mathbf{k}}{\left|\mathbf{x}_{2}\right|+\mathbf{x}_{2} \cdot \mathbf{k}}\right]
$$

where $\mathbf{x}_{i}$ denotes the heliocentric position vector of antenna $i$ and $\mathbf{k}$ the heliocentric unit vector towards the radio source. This time delay amounts to $\sim 170 \mathrm{nsec}$ for a source 
close to the limb of the Sun and $\sim 180^{\circ}$ away from the Sun. Since the total accuracy of the MARKIII VLBI system is of order $0.1 \mathrm{nsec}$, the solar gravitational time delay is seen in almost each individual measurement of the regular geodetic VLBI campaigns such as MERIT or POLARIS/IRIS. A first analysis of VLBI data through Oct. 84 from these projects gave a value for $\gamma$ of (Carter and Robertson 1985):

$$
\gamma=1.000 \pm 0.003 \quad \text { (formal standard error) }
$$

In a first step we reanalyzed a total of 54 IRIS experiments (Nov. 84 - Aug. 85) and found evidence for the Earth's gravitational time delay. More precisely, by multiparameter fit a multistation solution was obtained for each experiment with and without the effect for the gravitational time delay due to the gravitational field of the Earth. Here, $\tau_{\text {grav }}^{\oplus}$ can be expressed in terms of the antenna's elevation angles $E_{i}$ by

$$
\tau_{\mathrm{grav}}^{\oplus}=\left(\frac{\gamma+1}{2}\right) \frac{2 G m_{\oplus}}{c^{3}} \ln \left[\frac{1+\sin E_{1}}{1+\sin E_{2}}\right]
$$

and maximally is of order $0.02 \mathrm{nsec}$. On the average, including $\tau_{\mathrm{grav}}^{\oplus}$ reduced the residuals for the multistation solution by $1.58 \mathrm{psec}$. The statistical significance of this result is the subject further investigations. It is interesting to note that including $\tau_{\text {grav }}^{\oplus}$ into the fit program the baselines on the average changed by the amounts: Wettzell-Westford: $-0.5 \mathrm{~cm}$; Wettzell-Richmond: $\mathbf{- 2 . 2} \mathrm{cm}$; Fort Davis-Richmond: $+0.7 \mathrm{~cm}$ and Fort Davis-Westford: $+0.3 \mathrm{~cm}$.

After all, it seems that present VLBI techniques allow for a modern version of the old Gauss experiment and for a determination of the space curvature produced by the Earth.

*Research supported in part by the Deutsche Forschungsgemeinschaft (DFG), SFB78

\section{REFERENCES}

Carter, W., Robertson, D., MacKay, J., J. Geophys Res. 90, 4577.

Finkelstein, A., Kreinovich, V., Pandey, S., 1983, Astrophys. Space Sci. 94, 233.

Jones, B., 1976, Astron., J. 81, 455.

Reasenberg, R., Shapiro, I., MacNeil, P., Goldstein, R., Breidenthal, J., Brenkle, J., Cain, D., Kaufman, T., Komarek, T., Zygielbaum, A., 1979, Ap. J. 234, L219.

Soffel, M., Ruder, H., Schneider, M., Campbell, J., Schuh, H., 1986 in: Relativity in Celestial Mechanics and Astrometry, ed. by J. Kovalevsky and V. Brumberg, Reidel.

Texas Mauritanian Eclipse Team, Astron. J. 81, 452.

Will, C., 1981, Theory and Experiments in Gravitational Physics,, Cambridge University Press, Cambridge.

\section{DISCUSSION}

Eubanks: Two quick questions: what was the scatter in the Westford-Wettzell length changes under the relativistic correction, and what was your elevation angle cut-off?

Reply by Schuh: The variation was from about zero to $1 \mathrm{~cm}$, and the elevation angle cut-off was $10^{\circ}$. 